\title{
Dynamic calibration of piezoelectric transducers for ballistic high-pressure measurement
}

\author{
Lamine Elkarous $^{1,2,3, \star}$, Cyril Robbe ${ }^{1}$, Marc Pirlot $^{1}$, and Jean-Claude Golinval ${ }^{2}$ \\ 1 Royal Military Academy (RMA), Department of Weapons Systems and Ballistics, 30 avenue de la Renaissance, \\ 1000 Brussels, Belgium \\ 2 University of Liège (ULg), Department of Aerospace and Mechanical Engineering, 1-Chemin des Chevreuils, 4000 Liège, \\ Belgium \\ 3 Military Academy (AM), Fondouk-Jedid, Grombalia, 8012 Nabeul, Tunisia
}

Received: 3 December 2015 / Accepted: 6 March 2016

\begin{abstract}
The development of a dynamic calibration standard for high-amplitude pressure piezoelectric transducers implies the implementation of a system which can provide reference pressure values with known characteristics and uncertainty. The reference pressure must be issued by a sensor, as a part of a measuring chain, with a guaranteed traceability to an international standard. However, this operation has not been completely addressed yet until today and is still calling further investigations. In this paper, we introduce an experimental study carried out in order to contribute to current efforts for the establishment of a reference dynamic calibration method. A suitable practical calibration method based on the calculation of the reference pressure by measurement of the displacement of the piston in contact with an oil-filled cylindrical chamber is presented. This measurement was achieved thanks to a high speed camera and an accelerometer. Both measurements are then compared. In the first way, pressure was generated by impacting the piston with a free falling weight and, in the second way, with strikers of known weights and accelerated to the impact velocities with an air gun. The aim of the experimental setup is to work out a system which may generate known hydraulic pressure pulses with high-accuracy and known uncertainty. Moreover, physical models were also introduced to consolidate the experimental study. The change of striker's velocities and masses allows tuning the reference pressure pulses with different shapes and, therefore, permits to sweep a wide range of magnitudes and frequencies.
\end{abstract}

Keywords: Pressure measurement, dynamic calibration, high speed camera, acceleration and displacement

\section{Introduction}

Ballistics is in general the study of a projectile's motion in three-dimensional space. When a solid gun powder burns inside fired ammunition, a ballistic time-dependant high-pressure is generated and leads therefore to a physical dynamic quantity. The measurement of this so-called ballistic pressure is paramount. Indeed, the evaluation of gun and ammunition performances is closely related to the knowledge of this parameter. However, fast changes of its amplitude in a short period of time complicate the measurement which involves specific measurement techniques.

Unfortunately, the measurement of this quantity depends on the used measurement technique. Crusher, strain gauges and piezoelectric transducers are the most known techniques and continue to be used depending on their importance. Until mid of the 1960, the crusher gauge method, invented in 1860 by Andrew Nobel was

\footnotetext{
* Correspondence: walktohunt1982@gmail.com
}

the commonly used method. Since the development of the charge amplifiers by Kistler in the 1950s, the piezoelectric transducers became the main technique and knew a great progress in ballistic high-pressure metrology [1] Indeed, sensors with range over 1 kbar are called highpressure sensors. Their main applications are in highpressure hydraulics, in particular fuel injection pumps of diesel engines and ballistics.

Depending on the weapon's caliber, the whole ballistic phenomenon takes only a few milliseconds when ammunition is fired. Regardless to this fact, the calibration techniques of the used piezoelectric transducers are still mainly static (dead-weight) or quasi-static (continuous). There is no adequate primary dynamic standard for ballistic transducers due to the absence of an absolute generator which can provide the reference dynamic pressure. An improper calibration certainly leads to incorrect measurement and increases the uncertainty. Hence, bad lots of ammunition can be accepted and good lots can be rejected. The safety of the use of a gun could also decrease if the maximum pressure is not known precisely. 
Thereby, the need of a reliable dynamic calibration technique for such transducers has been increasing, particularly in the last twenty years. Many methods have been developed and employed to calibrate the variety of pressure sensors: shock tube, quick-opening device, explosive devices and the drop-weight devices [2,3]. But noone of them was able to satisfy entirely the need for ballistic highpressure piezoelectric transducers. The reasons for this limitation is the range of amplitude (hundreds of $\mathrm{MPa}$ ) and frequency (tens of $\mathrm{kHz}$ ) of the gas pressure signal. They are all aperiodic generators which create step-like pressures or single pressure pulses similar to a half-sine wave. In most cases, the reference pressures are measured by reference transducers from the same categories and calibrated statically [3].

The present study falls within this topic with the aim of developing a dynamic calibration method. A suitable practical calibration method based on the calculation of the reference pressure by the measurement of the displacement of the piston in contact with an oil-filled cylindrical chamber is presented. This measurement was achieved thanks to a high speed camera or an accelerometer. Both measurement are then compared. In the first way, pressure was generated by impacting the piston with a free falling weight and, in the second way, with strikers of knows masses accelerated to the impact velocities with an air gun. The aim of the experimental setup is to work out a system which may generate known hydraulic pressure pulses with high-accuracy and controlled uncertainty. Moreover, a mathematical description based on different physical models is also introduced to consolidate the experimental method.

\section{Dynamic pressure calibration}

\subsection{A Survey of the methods}

The work of Schweppe et al. published in 1963 is considered the first reference document in the field of dynamic calibration of pressure sensors [4]. In 1972, ASME (American Society of Mechanical Engineers) published a guide for the dynamic calibration of pressure sensors [5]. A revised version of this guide by ISA (Instrumentation, Systems, and Automation Society) has been available since 2002 [6]. This second document consists of an elaborate version of the first one, where the pressure sensors properties and the dynamic calibration methods were presented more in detail. The diversity of periodic and aperiodic generators which have been developed was described. Each pressure generator has its range of operation. Periodic generators give pressures with low amplitudes and limited bandwidths, while aperiodic generators produce pressures with high amplitudes and large bandwidths. The choice of the required device is then determined by the conditions of use of the sensor to be calibrated. However, it should be noted that the transient and the harmonic tests are theoretically equivalents and allow both to characterize the dynamic behavior of the sensor [3].
The pressure standards under static conditions, as well as the associated uncertainties, are characterized precisely. The pressure balance is a primary standard that can provide highly accurate reference pressures with relative uncertainty of about $0.012 \%$. This method can only provide the sensitivity and linearity of a piezoelectric pressure sensor but does not provide any information on its dynamic behavior either in time and frequency domains [7].

A pressure primary standard for the dynamic calibration of high-pressure sensors does not exist yet. Indeed, the pressure signals provided by the existing aperiodic generators, especially shock tubes and quick-opening devices, are not perfectly known in amplitude and frequency except through a statically calibrated reference sensor. The shock tube may be an exception because the amplitude of the reference step signals can be calculated based on thermodynamic models. However, it requires precise knowledge of the gas properties and the shock wave speed in the tube. For this reason, a reference sensor is always used although the research aims to develop a shock tube primary standard. Few national institutes of metrology have developed series of shock tubes but they are still limited essentially for high-amplitude pressure. Indeed, the shock tube is very useful to study the dynamic behavior of the pressure sensors at high frequencies (usually up to $100 \mathrm{kHz}$ ), but the amplitude of the pressure remains limited to about $10 \mathrm{MPa}$ which is still lower than most ballistic peak pressures [8]. Quick-opening devices generate positive or negative steps. A device generating amplitude pressure levels of about $1 \mathrm{GPa}$ and a rise time less than $1 \mathrm{~ms}$ has been developed to calibrate high-pressure transducers [2]. In most cases, the frequency bandwidth of these devices is still limited.

Moreover, the concept of dynamic pressure generation by use of the close vessel has long been known. Mickevicz developed a bomb for generating pressures of about $300 \mathrm{MPa}$ at the Naval Ordnance Laboratory [4]. This class of dynamic pressure generators which employs the burning of a gun powder has also been reviewed by Damion [3]. However, the lack of accuracy on the determination of the peak pressure, the high-rise time, the danger especially at high pressure and the time consuming handling of a single test were sufficient reasons to make this method rather unsuitable for dynamic pressure calibration. Nevertheless, some institutes continue to use it based on a so-called reference powder for dynamic tests on pressure transducers.

For ballistic high-pressure transducers, the only available method nowadays is static calibration. A dynamic verification or adjustement was also introduced to improve their behavior for dynamic measurements as in weapon barrels and closed vessels. First of all, only the working standard is calibrated statically by the use of a pressure balance as the primary standard (step-wise calibration). Then, a quasi-static calibration of the piezoelectric transducers is fulfilled by a high-continuous pressure generator as a routine calibration using that working standard. Finally, their dynamic behavior is checked against the same working standard [9]. For this purpose, the pressure pulse 
generators with free-falling weights are widely used due to the absence of a primary dynamic calibration system. When the drop weight hits a piston in contact with an oil-filled chamber, the generated pressure pulse is similar to a half-period sine; its amplitude depends on the compressibility of the fluid, the weight, the drop height and the piston section. The reference pressure is still unknown and also no-indications about the temperature and the pressure waves are given. This last is assumed to be perfect and both sensors, sensor under test (SUT) and reference sensor (RS), mounted in opposing ports are subject to the same wave.

However, differences between peak pressures and between ascending and descending phases, inherent to hysteresis phenomena, can be observed. Thus, based on the peak pressure given by the two sensors, a so called dynamic sensitivity is calculated. In the other way, a polynomial fitting is carried out to achieve an adjustment operation between the SUT and the RS. The polynomial degree has to be chosen carefully by comparing pressuretime signals. Hence, the pressure pulse generator is still used for a comparison calibration based on a reference sensor which provides the reference pressure measurements. In general, this generator has been adapted to get a pressure curve pattern quite similar to real gas pressure variation inside fired ammunition; both in its duration and in its shape. The aim of this method is to check that the dynamic behavior of the pressure transducer can mimic the real gas pressure which certainly improves the accuracy of the measurement. For almost all available systems such as those developed by HPI GmbH [7], Kistler AG [9], PTB [10] and PCB Piezotronics [11] sinusoidal pressure pulses are characterized with widths from 3 to $7 \mathrm{~ms}$, rise times of about $2 \mathrm{~ms}$ and peak pressures up to $800 \mathrm{MPa}$.

The last researches in the field of dynamic highpressure calibration were conducted in the works of Bartoli et al. [12], Bruns et al. [13] and Zhung et al. [14]. The goal of the studies was to establish traceable dynamic measurement of the mechanical pressure. In the first and the second work, which are made in the same EMRP framework, the reference pressure is deduced from laser interferometry and accelerometer measurements of the deceleration of drop-weight during impact or by laser interferometry measurements of the density change via the refractive index change in the pressurized media. However, in the third work, the Hopkinson bar principle was used to generate high-amplitude pressure pulses under highly-static conditions. The input excitation was measured using a strain gauge and the frequency response function of the pressure sensor was easily computed.

\subsection{Calibration principle}

Calibration is a procedure to find the relationship between a known input $e(t)$ and a measured output $s(t)$ in welldefined conditions, which means the determination of the transfer function $[2-4,6]$. The amplitude of this function represents the change in the sensitivity as a function of the frequency, so that its phase is used to find the phase shift between the output signal of the data sensor and a reference pressure ideally known. In static calibration, the ratio of output and input is constant and gives the sensitivity of the sensor. In dynamic calibration, the ratio leads to a complex function $H(\omega)$ which is defined as the ratio of the Fourier transforms of the output $S(\omega)$ and the input $E(\omega)[3-6]$ :

$$
H(\omega)=\frac{S(\omega)}{E(\omega)}=\frac{\int_{0}^{\infty} s(t) e^{-j \omega t} d t}{\int_{0}^{\infty} e(t) e^{-j \omega t} d t} .
$$

In practice, it's impossible to determine the characteristics of the pressure transducer analytically. Therefore, the transfer function is estimated from a knowledge of a pair of associated input and output signals $e(t)$ and $s(t)$ which are derived from the responses of the complete measuring chain (sensor, charge amplifier, data acquisition and processing system). The measured signals are sampled and non-periodic. Discrete Fourier transform (DFT) is then used to compute the transfer function. The transfer function allows the full characterization of pressure sensors both in static and in dynamic. Besides the transfer function as amplitude response (AFR) and phase response (PRF), several properties of the pressure sensor such as resonance frequency, damping ratio, rise time and overshoot have to be assessed [6].

\section{Dynamic description of ballistic pressure transducers}

Piezoelectric ballistic pressure transducers are typically modeled by a single degree of freedom oscillating linear system. The model consists of at least a mass $m(\mathrm{~kg})$, a spring with a spring constant $k(\mathrm{~N} / \mathrm{m})$ and a damper with damping constant $c(\mathrm{~N} . \mathrm{s} / \mathrm{m})[6]$. That is, the oscillatory movement would be limited to a direction $(x)$ parallel to the line of action of the applied force $f(t)$. The characteristic differential equation which describes the motion of the system is obtained by application of d'Alembert's principle. When rearranged onto its canonical form, we get:

$$
\frac{d^{2} x(t)}{d t^{2}}+2 \xi \omega_{n} \frac{d x(t)}{d t}+\omega_{n}^{2} x(t)=\frac{f(t)}{m}
$$

where, $\omega_{n}=\sqrt{\frac{k}{m}}(\mathrm{rad} / \mathrm{s})$ is the natural frequency of the system and $\xi=\frac{c}{2 \sqrt{k m}}$ is the damping ratio. Applying the Laplace transform to equation (2) yields to the transfer function of the pressure sensor:

$$
H(s)=\frac{X(s)}{F(s)}=\frac{K \omega_{n}^{2}}{s^{2}+2 \xi \omega_{n} s+\omega_{n}^{2}}
$$

where, $K(\mathrm{pC} / \mathrm{MPa})$ is the steady-state sensitivity and $s=j \omega=j 2 \pi f$ is the complex variable ( $f$ is the frequency). Using the expression of the transfer function, the inverse Laplace transform is then applied to determine the time-domain response of the sensor to any input force (step, pulse or sine functions) can be obtained. 
Both the step and the impulse responses characterize the dynamic behavior of transducers as the impulse response is the derivate of the step response. If we apply a pressure step of amplitude A to the transducer, the time response is:

$$
\begin{aligned}
x(t)= & A\left[1-\frac{1}{\sqrt{1-\xi}} e^{\left(\frac{\xi \omega_{d} t}{\sqrt{1-\xi^{2}}}\right)}\right. \\
& \left.\times \sin \left(\omega_{d} t+\operatorname{arct}\left(\frac{\sqrt{1-\xi^{2}}}{\xi}\right)\right)\right] .
\end{aligned}
$$

Here, $\omega_{d}=\omega_{n} \sqrt{1-\xi^{2}}$ is the damping frequency.

\section{Experimental study of the pressure pulses generator}

\subsection{The high pressure generator}

A hydraulic pressure pulse generator Kistler type 6909 has been used for the assessment of the ballistic pressure transducers performance in a dynamic environment. The device consists of a piston/cylinder manifold and a drop tube containing a mass that can be dropped onto the piston from various heights [1]. When the mass is released from a certain height along the guiding tube, it transmits its Kinetics energy through the piston to the fluid inside the hard steel structure. The collision is perfectly inelastic. For that, the drop weight and the piston move downward with the same velocity. At the same time, the piston skins rapidly into the oil-filled chamber into which the pressure transducers are mounted. Its energy is then transformed to the fluid as compression energy which causes a pressure increase.

After releasing the total energy, the maximum pressure is reached and the reverse motion of the piston and drop weight starts. They are pushed upwards with the same velocity until the piston is stopped and the falling mass rebounds, and is generally caught. During this process, a pressure pulse is generated, which is similar to a single half cycle of a sine wave. Its amplitude depends on the fluid bulk modulus $K(\mathrm{MPa})$, the total mass (falling mass $m_{t}$ and piston $\left.m_{p}\right)$, the height $h(\mathrm{~m})$ and the piston area $A$ $\left(\mathrm{m}^{2}\right)$. The ultimate goal is to obtain a pressure-time signal that mimics the dynamics of weapon chamber pressures.

The principle of the calibration mode is depicted in Figure 1 below. The whole system was also modeled by a mass $m(\mathrm{~kg})$, a spring with a spring constant $k(\mathrm{~N} / \mathrm{m})$ and a damper with damping constant $c(\mathrm{~N} . \mathrm{s} / \mathrm{m})$. The generated pressure $p(t)$ is proportional to the displacement of the total mass $x(t)$.

The velocity of the free falling mass just prior the impact was calculated considering only the acceleration due to the gravity $g\left(\mathrm{~m} / \mathrm{s}^{2}\right)$ :

$$
v_{f}=\sqrt{2 g h} .
$$

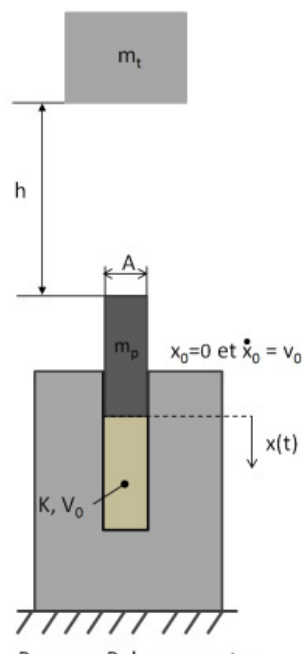

Pressure Pulse generator

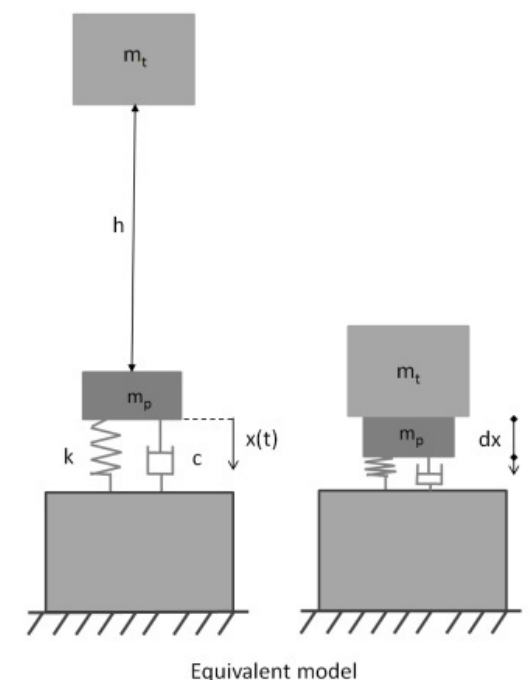

Fig. 1. The hydraulic pressure pulse generator.

During the impact, the collision was assumed perfectly inelastic i.e. the falling mass does not bounce but becomes permanently attached to the piston mass. In addition, the velocity $v_{0}$ of the total mass $m=m_{t}+m_{p}$ immediately after the impact was obtained using the conservation of momentum. Given that the mass of the piston $\left(m_{t}=0.006 \mathrm{~kg}\right)$ can be neglected comparing to the falling mass $\left(m_{p}=5.4 \mathrm{~kg}\right)$, we get:

$$
v_{0}=\frac{m_{t}}{m_{t}+m_{p}} v_{f} \approx v_{f}
$$

\subsection{Measurement and calculation of the reference pressure}

In this study, the dynamic behavior of the pressure transducer will be assessed using the peak pressure values. However, the dynamics of the phenomenon will also be taken into account. The reference pressure calculation is based on the measurement of the deceleration and the displacement of the mass, during the compression and the expansion phases of the process. This measurement is performed thanks to a high speed camera and an accelerometer. Both measurements are performed in parallel, allowing a cross validation of both principles. High speed cameras have become widely used to measure dynamic phenomena such as in ballistics. This versatile technique has the advantage of the simplicity of handling, set-up and mainly no modification of the measuring system is required.

For the pressure pulses generator depicted in Figure 1, expression of the conservation of mechanical energy before and after the falling mass hits the piston yields to:

$$
m_{t} g h=\frac{1}{2} m v^{2}+\int_{0}^{x} p A d x
$$

where, $v$ is the velocity of the total mass after the impact. Therewith, when all the potential energy is transmitted 
to the fluid as compression energy, we get:

$$
m_{t} g h=p_{\max } A x_{\max } \Leftrightarrow p_{\max }=\frac{m_{t} g h}{A x_{\max }}
$$

where, $p_{\max }$ is the maximum pressure and $x_{\max }$ is the maximum displacement of the piston.

In addition, the process is considered as isothermal. The isothermal bulk modulus $K(\mathrm{MPa})$ given by equation (9) was thereby used as approximation to compute the pressure variation caused by the change in volume.

$$
K=-d p \frac{V}{d V}=d p \frac{\rho}{d \rho}
$$

Hence, a direct link can be made between the pressure and the displacement as follows:

$$
d p=-K \frac{d V}{V}=K \frac{A d x}{V_{0}-A x} \Longrightarrow p=\int_{0}^{\mathrm{x}} K \frac{A}{V_{0}-A x} d x
$$

This method was also used at MIKES within the EMRP framework [12]. The obtained results of displacement and pressure were compared to the ones deduced from the equivalent mass-spring-damper model (EM) illustrated in Figure 1. The equation of motion of the total mass is described by the following second order differential equation.

$$
m \frac{d^{2} x(t)}{d t^{2}}+c \frac{d x(t)}{d t}+k x(t)=m g .
$$

For the initial conditions $t=0, x(0)=0, \dot{x}(0)=v_{0}$, the solution of this equation is given by:

$$
x(t)=a e^{-\delta t} \sin \left(\omega_{d} t+b\right)+\frac{m g}{k}
$$

where

$$
\begin{aligned}
& \text { - } \delta=\xi \omega_{n} \\
& \text { - } a=\frac{v_{0}}{\omega_{d} \cos (b)-\delta \sin (b)} \\
& \text { - } b=\operatorname{arctg}\left(\frac{\omega_{d} g}{\delta g-v_{0}\left(\omega_{d}^{2}+\delta^{2}\right)}\right) .
\end{aligned}
$$

Figure 2 gives an overview of the experimental set-up which was used to measure the deceleration and the displacement of the falling weight and the piston.

An ICP shock accelerometer PCB type 305A03 with sensitivity equal to $0.477 \mathrm{mV} / \mathrm{g}$ was mounted adhesively onto the top of the drop weight. Moreover, the high speed camera was installed horizontally at a defined distance and level to get an optimal angle of view which permits to follow the whole collision process as shown in Figure 3.

The used camera is a Photron SA-5 high-speed camera (HSC). This HSC can achieve a frame rate up to one million frames per second (fps) [15]. For the calculation, the body formed by the piston and the drop weight is assumed to be rigid during the collision process and its movement is

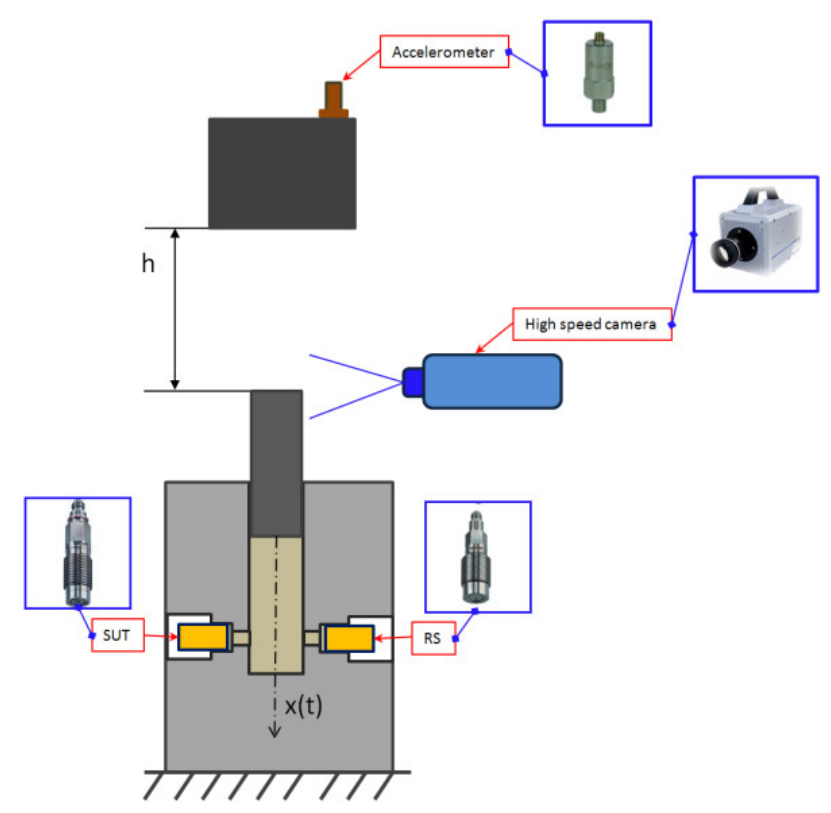

Fig. 2. The experimental set-up of the pressure pulse generator.

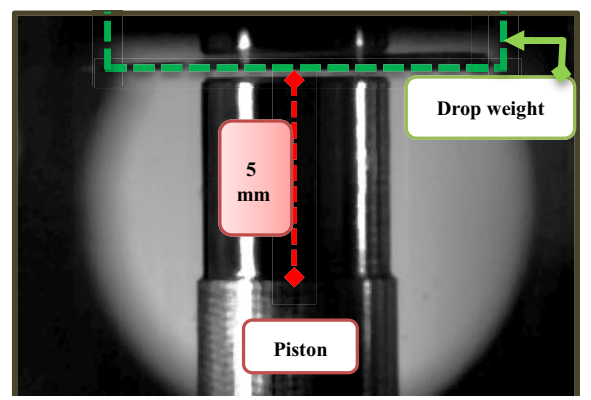

Fig. 3. A photograph of the process by HSC prior to the impact.

also supposed to be perfectly straight, always in the same plan of the camera axis.

The frame rate was set at $50000 \mathrm{fps}$. The calibration factor is 29.2 pixels $/ \mathrm{mm}$. In addition, taking into account the small dimensions of the piston and the lack of space in the measurement zone, a special lens with $12 \mathrm{X}$ magnification was used. Also, special attention was devoted to the problem of lighting to ensure a high quality measurement. A thorough explanation of the velocity computing based on the HSC measurement was performed by Robbe et al. [16].

The piezoelectric pressure transducers Kistler type 6215 (DUT) and type 6213BK (RS) delivers low amplitude and high impedance charge signal, generally expressed in pico-Coulomb (pC). Thus, a charge amplifier Kistler type $6907 \mathrm{~B}$ with scale factor of $100 \mathrm{MPa} / \mathrm{V}$ was used. The data acquisition (DAQ) board consists in a multi-channel device of four high speed digitizers. Each digitizer has two channels of parallel with a resolution of 14 bits and a maximum sampling rate of $100 \mathrm{MHz}$. The major conversion occurring in the DAQ board is an analog to digital conversion. 


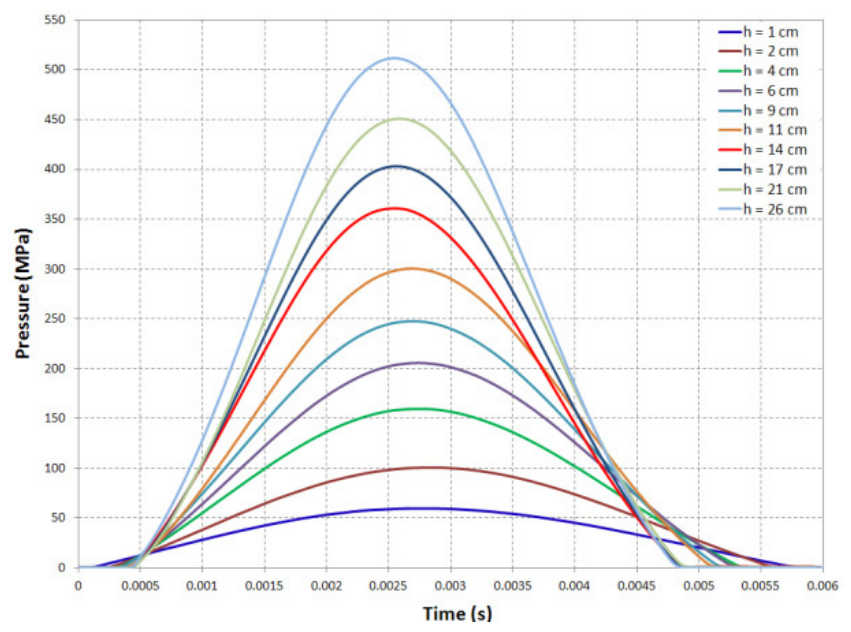

Fig. 4. Pressure-time curves for different heights.

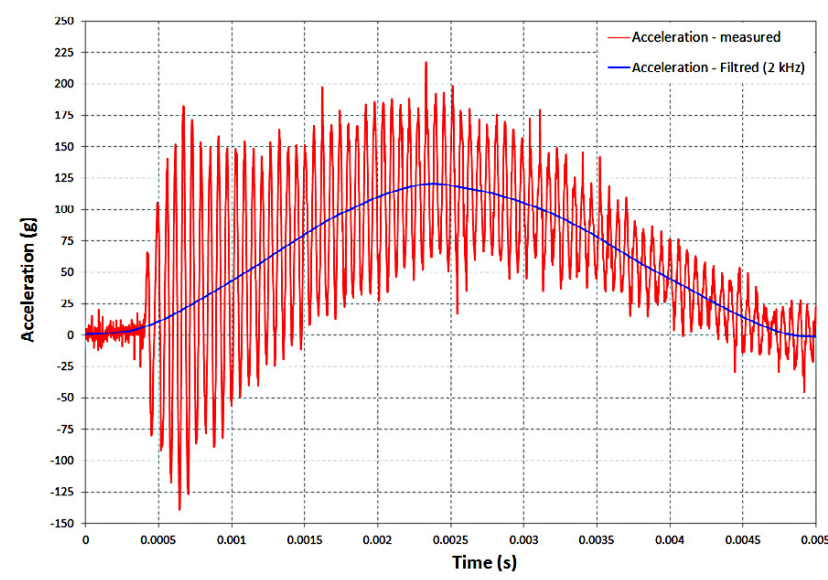

Fig. 5. Acceleration of the piston during the process.

In addition, signal processing was performed with programs developed with the LabVIEW software [17]. A Butterworth phaseless filter was used. For all signals, the frequency domain transform was computed by DFT for optimizing the filter cut off to the signal.

\subsection{Results and discussions}

Test series of 10 measurements for 10 chosen drop weight heights have been made with the experimental bench described in Figure 2. Figure 4 below shows typical pressuretime curves given by the hydraulic pulse generator for different selected heights.

The pressure pulses are characterized by a width of about $5 \mathrm{~ms}$ and a rise time of about $2 \mathrm{~ms}$. The maximum pressure varies from 60 to $510 \mathrm{MPa}$.

Similarly, Figure 5 illustrates a typical measured deceleration of the falling weight and piston during the collision process.

When the drop weight strikes the piston, an elastic wave is created directly and is transmitted into the piston. This wave is also reflected in the drop weight.

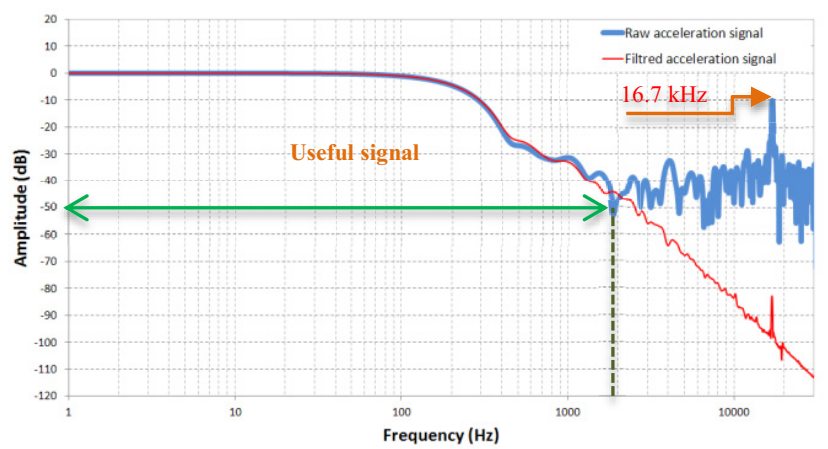

Fig. 6. FFT of the acceleration-time raw and filtered signals.

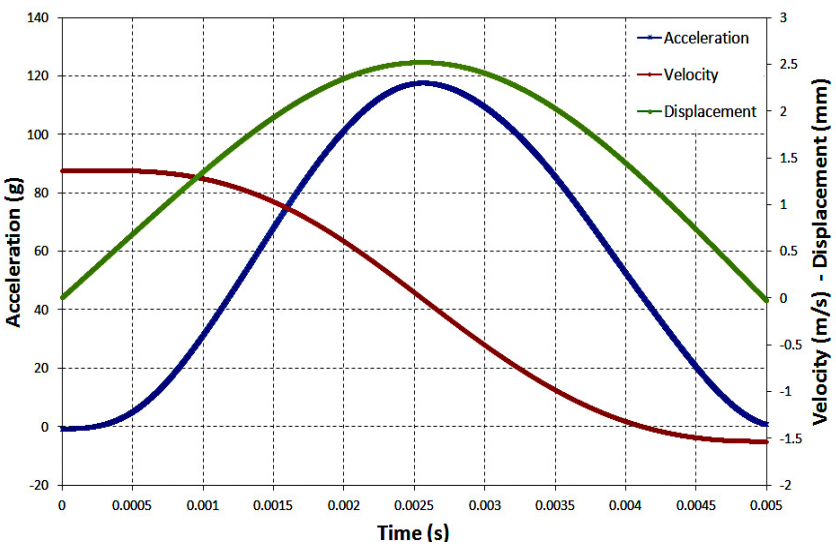

Fig. 7. Filtered acceleration, velocity and displacement.

As the falling weight and the piston remain attached during the whole compression and expansion process, the to-and-fro motion of the wave leads to the oscillations observed on the accelerometer signal in Figure 5. The amplitude of the reflected wave is also more important at the compression phase of the process but it decreases progressively at the expansion phase. The period of the oscillations is constant and is around $60 \mu \mathrm{s}$. This period corresponds to the duration $\left(T_{c}\right)$ required for the wave to travel twice the drop weight length $\left(L_{d w}=0.15 \mathrm{~m}\right)$ which is given equation (13). The speed of the wave $c(\mathrm{~m} / \mathrm{s})$ is $5020 \mathrm{~m} / \mathrm{s}$.

$$
T_{c}=\frac{2 L_{d w}}{c} .
$$

In addition, the Fast Fourier Transform (FFT) was used to investigate deeply the frequency content of the accelerometer signal.

First of all, as can be seen in Figure 6, there is peak around $16.7 \mathrm{kHz}$ which matches the frequency of the oscillations as it was determined by equation (13). Secondly, it can also be noticed that the useful signal is mostly below the frequency of $2 \mathrm{kHz}$.

The velocity and the displacement of the piston and drop weight assembly, when it moves downward and upward after the collision, are obtained by the integration of the filtered acceleration-time signal. Figure 7 illustrate the three signals for a drop height of $14 \mathrm{~cm}$. 


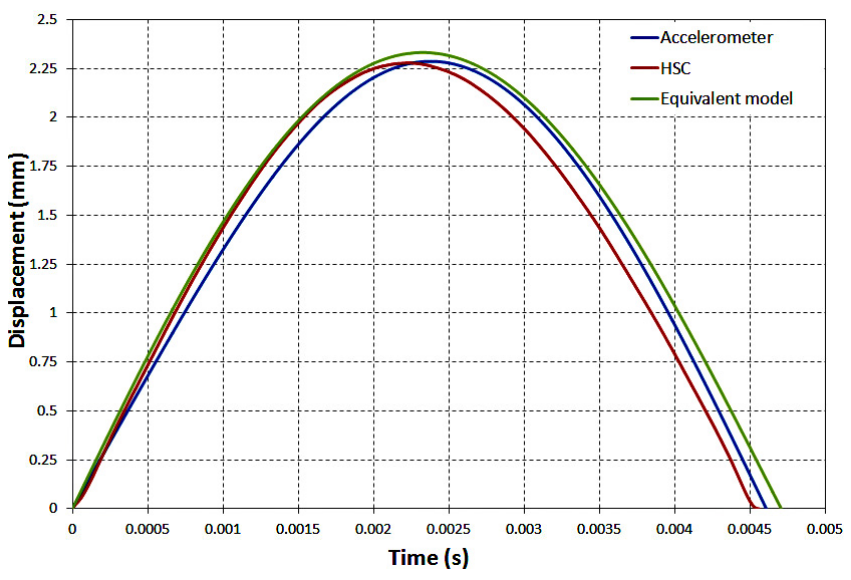

Fig. 8. Displacement: accelerometer, HSC and equivalent model.

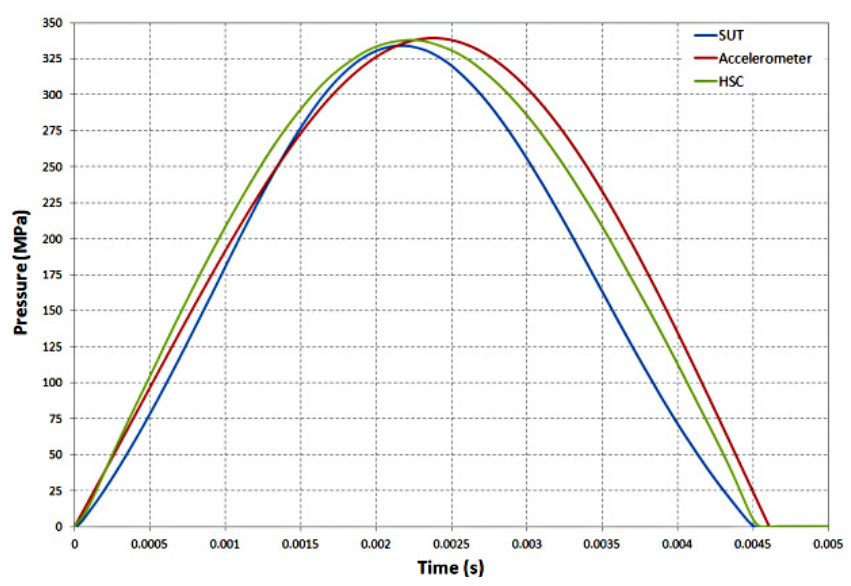

Fig. 9. Pressure inside the compression chamber.

The shape of the displacement time-signal is in good accordance with the pressure curves presented in Figure 4. In Figure 8, the displacements given by the accelerometer, the HSC and the equivalent mechanical model (EM) are plotted together.

The partial conclusions are:

- The shapes of the time-displacement curves are similar.

- The peak values and duration are slightly different, which will be evaluated later.

- The proposed equivalent model fits the experimental results and can predict reliably the motion of the piston during the whole process.

- The displacements given by the HSC and the accelerometer are practically identical and always lower than the theoretical displacements.

Using the model described by equation (10), the pressure inside the oil-filled chamber was then computed as shown in Figure 9.

Comparing the pressure-time signals given by the accelerometer and the HSC to the one measured by the SUT, peak value and duration of the process can be determined.

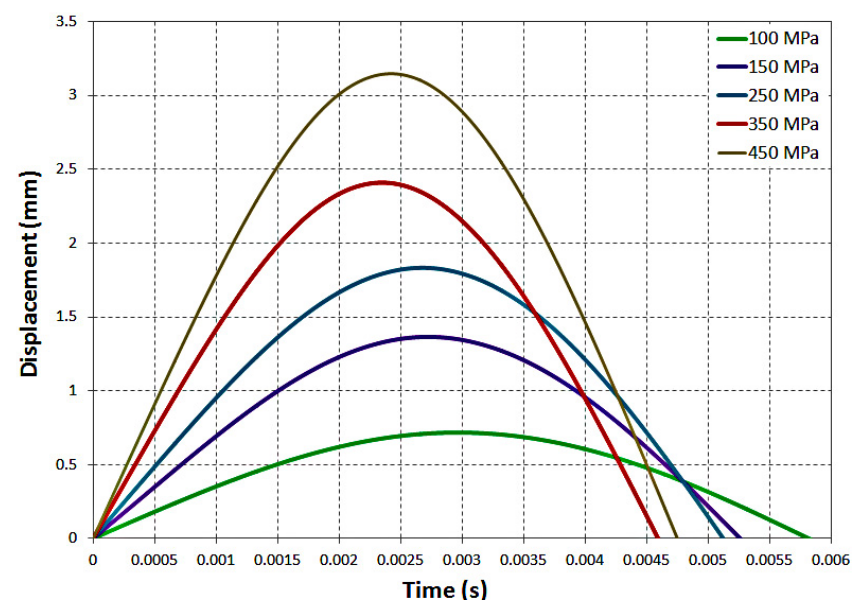

Fig. 10. Displacements measured by the accelerometer.

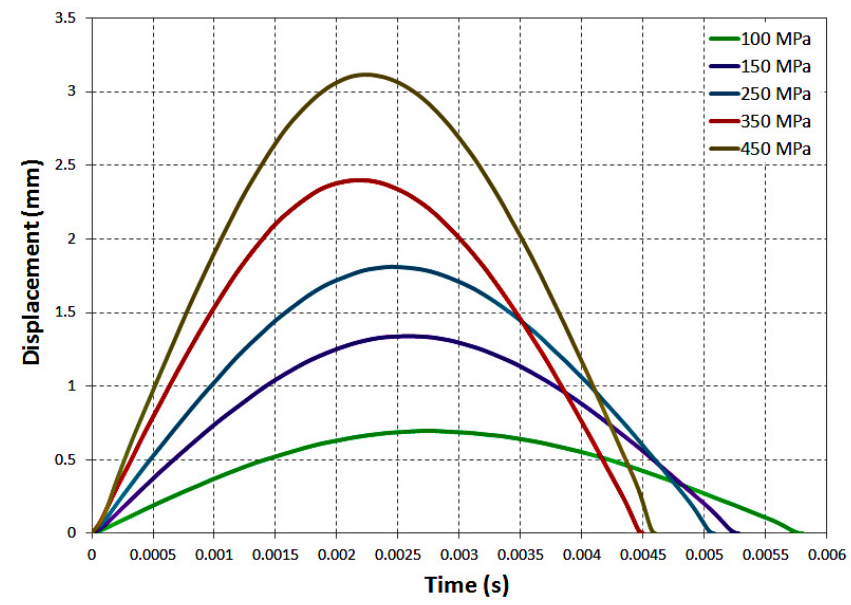

Fig. 11. Displacements measured by the HSC.

Figures 10 and 11 give the measured displacement for some selected heights of the drop weight.

The time-displacement curves of the drop weight/ piston given by the accelerometer and the HSC for different falling heights are fairly similar.

In order to evaluate the two measuring methods, the peak values were taken as comparison criterion. Table 1 summarizes the measured maximum values of the displacement as well as for the pressure.

The maximum values of displacement were therefore used to calculate the maximum pressure $\left(P_{\max }\right)$, i.e. the reference pressure $\left(P_{\text {ref }}\right)$, using equation $(14)$ which was derived from equation (10):

$$
P_{\max }=-K \ln \left(1-\frac{A x_{\max }}{V_{0}}\right) .
$$

Comparing the maximum pressure between each other, the obtained relative difference in $(\%)$ is presented in Figure 12.

Despite the fact that the accelerometer always gives maximum pressure values slightly greater than the HSC, good agreement was observed between the two measuring techniques. The relative difference between the maximum 
Table 1. Maximum values of displacement and pressure.

\begin{tabular}{cccccccc}
\hline \multirow{3}{*}{$h(\mathrm{~cm})$} & \multicolumn{3}{c}{$x_{\max }(\mathrm{mm})$} & \multicolumn{5}{c}{$P_{\max }(\mathrm{MPa})$} \\
\cline { 2 - 7 } & Accelerometer & HSC & $\begin{array}{c}\text { Rel. } \\
\text { error }(\%)\end{array}$ & Accelerometer & HSC & $\begin{array}{c}\text { Rel. } \\
\text { error }(\%)\end{array}$ & RS \\
\hline 1 & 0.511 & 0.503 & 1.57 & 65.75 & 64.71 & 1.59 & 60.83 \\
2 & 0.821 & 0.810 & 1.34 & 106.78 & 105.31 & 1.38 & 101.60 \\
4 & 1.257 & 1.241 & 1.27 & 166.03 & 163.82 & 1.33 & 160.01 \\
6 & 1.593 & 1.572 & 1.32 & 213 & 210.01 & 1.39 & 207.90 \\
9 & 2.035 & 2.013 & 1.08 & 276.56 & 273.35 & 1.16 & 270.50 \\
11 & 2.270 & 2.250 & 0.88 & 311.26 & 308.28 & 0.96 & 303.17 \\
14 & 2.608 & 2.597 & 0.42 & 362.30 & 360.61 & 0.46 & 357.75 \\
17 & 2.929 & 2.918 & 0.38 & 412.07 & 410.34 & 0.42 & 407.20 \\
21 & 3.186 & 3.176 & 0.31 & 452.90 & 451.27 & 0.35 & 450.24 \\
26 & 3.466 & 3.461 & 0.14 & 498.36 & 497.54 & 0.16 & 495.05 \\
\hline
\end{tabular}

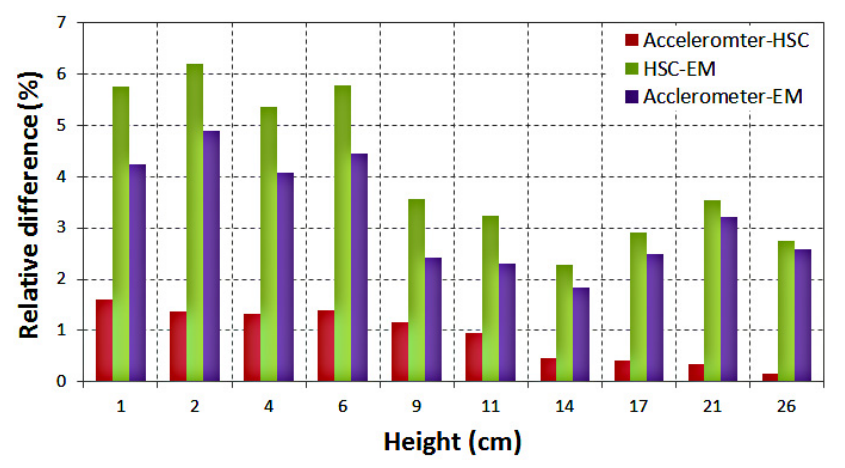

Fig. 12. Relative difference of maximum pressure.

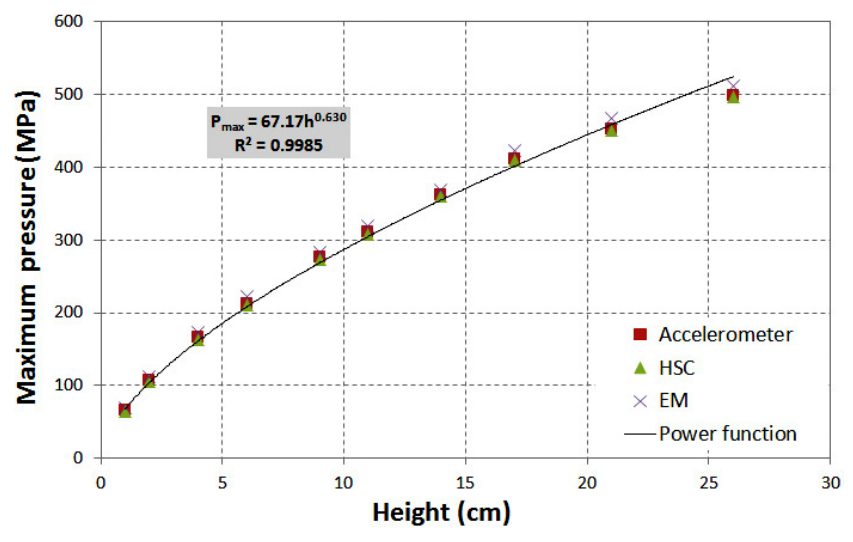

Fig. 13. Maximum pressure versus falling height.

pressures remains generally below $1 \%$. The equivalent model overestimates the maximum pressure with a relative difference almost exceeding the $3 \%$ and could easily reach $6 \%$ compared to the two measuring methods. Nevertheless, this estimation remains acceptable and leads to consider the physical model well enough to be used to predict the maximum displacement and pressure.

Fitting the maximum pressure to the height of falling weight with the power function, a relationship could be obtained as shown in Figure 13.

According to the described experiments, it follows that the measurement methods of the displacement, by an accelerometer or an HSC, are equivalent.

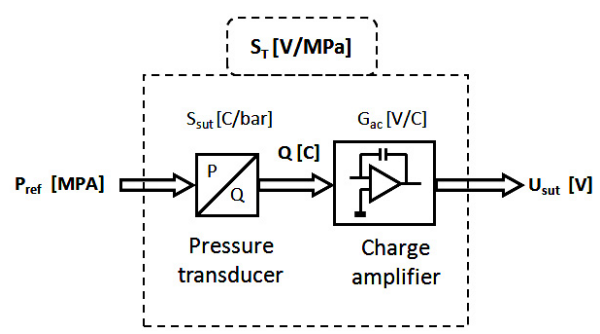

Fig. 14. The model of the measuring chain.

The simultaneous measurement certainly improves the results. However, only the HSC was used to achieve the defined purpose especially as the HSC measurement uncertainties could be computed [16].

\subsection{Dynamic sensitivity of the piezoelectric pressure transducer}

As it was already mentioned, the measuring chain is constituted, in addition to the pressure transducer, of a charge amplifier, a data acquisition module and signal processing unit.

Considering the sketch in Figure 14, the sensitivity of the sensor $S_{\text {sut }}(\mathrm{pC} / \mathrm{MPa}$ ) to be calibrated (SUT) could also be computed by the following relationship:

$$
S_{\text {sut }}=\frac{Q}{P_{\mathrm{ref}}}=\frac{U_{\mathrm{sut}}}{P_{\mathrm{ref}} G_{a c}}
$$

where $U_{\text {sut }}(\mathrm{V})$ denotes the maximum voltage delivered by the whole measuring chain of the SUT, $P_{\text {ref }}(\mathrm{MPa})$ is the reference pressure which outcomes from the measurement of the HSC and $G_{a c}=\frac{U}{Q}(\mathrm{~V} / \mathrm{pC})$ is the gain of the charge amplifier. Practically, this gain was measured using a precision capacitor of $1000 \mathrm{pF}$. An AC voltage source was employed to provide voltage signals with different frequencies to the charge amplifier by way of the mentioned capacitor. Applying a voltage $U$ across the $1000 \mathrm{pF}$ capacitor is effectively applying U.1000 pC of charge into the charge amplifier $(Q=U C)$. Figure 15 illustrate a typical amplitude frequency response of the charge amplifier. 


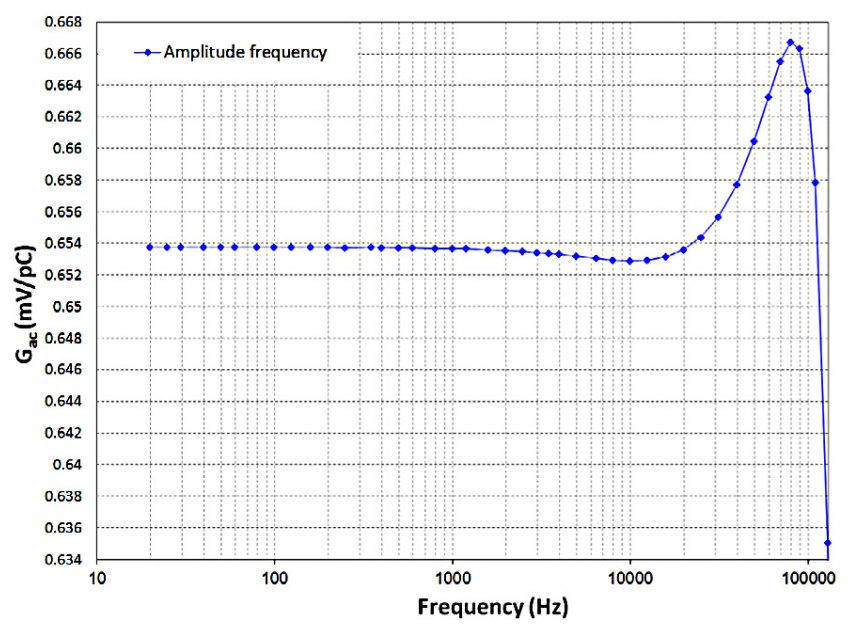

Fig. 15. A typical amplitude frequency of the charge amplifier.

The dynamic measurements are carried out with a short time constant.

The gain of the charge amplifier in the frequency range up to $25 \mathrm{kHz}$ showed no significant deviations. There is only a relatively small deviation of about $0.1 \%$. In the range up to $110 \mathrm{kHz}$, the relative deviation remains under $2 \%$.

\subsection{Assessement of the calibration uncertainty}

According to the Guide to the Expression of Uncertainty in Measurement (GUM) published by ISO in 1993 [18], the combined-standard uncertainty of measurements includes two general terms: one depends on the number of observations $\left(u_{A}\right.$, type $\mathrm{A}$ uncertainty), and the other not $\left(u_{B}\right.$, type B uncertainty), and can be expressed as follows:

$$
u_{c}=\sqrt{u_{A}^{2}+u_{B}^{2}}
$$

where $u_{A}=s(\bar{x})=\frac{s}{\sqrt{n}}$ which is also defining the repeatability error and $s$ is the experimental standard-deviation of a number of observations $n$. The estimators of the mean peak and standard deviation pressure value are given by:

$$
\bar{x}=\frac{1}{n} \sum_{i=1}^{n} x_{i} \quad \text { and } \quad s=\sqrt{\frac{1}{n-1}} \sum_{i=1}^{n}\left(x_{i}-\bar{x}\right)^{2} .
$$

In addition, $u_{B}$, the uncertainty of measurement due to different sources of independent uncertainty, is expressed by equation (18):

$$
u_{B}=\sqrt{\sum_{i=1}^{n}\left(\frac{\partial f}{\partial x_{i}}\right)^{2} u^{2}\left(x_{i}\right)}=\sqrt{\sum_{i=1}^{n} u_{B i}^{2}}
$$

where $f$ indicates the measurement which is also considered as a function of different parameters $x_{i}$ and $u\left(x_{i}\right)$ is the equation of an individual uncertainty driven by the parameter $x_{i}$. Here, the elementary uncertainties $u\left(x_{i}\right)$ are supposed to be independent and approximately linear.

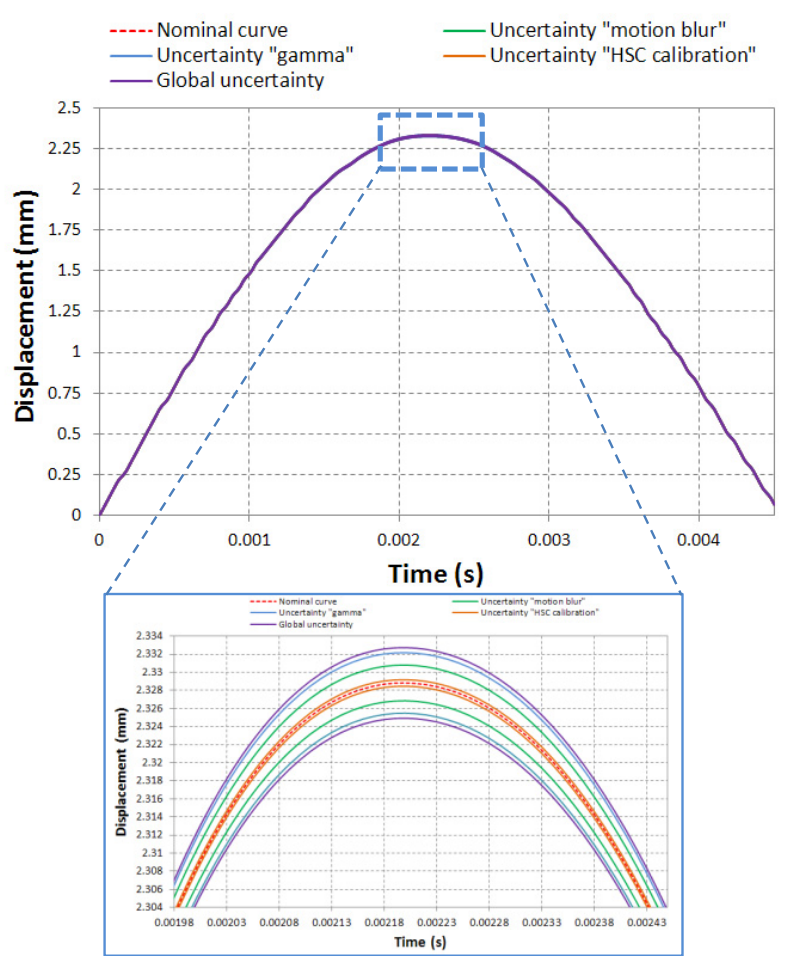

Fig. 16. Measurement uncertainty of displacement.

As mentioned above, the calculation of the displacement and its measurement uncertainty were performed by a dedicated program [16]. Individual uncertainties were computed and the global uncertainty was determined precisely. Figure 16 shows the displacement of the drop weight and piston during the collision as a function of time for a chosen falling height of $13 \mathrm{~cm}$. By considering three sources of errors, the uncertainty computations allow to obtain a corridor around the nominal displacement curve in which the real measurement should exist.

The shape of the corridor changes along the curve due to the change of the individual contributions of all the uncertainties. Here, the global relative uncertainty on the maximum value is equal to $0.17 \%$ which corresponds to a combined-standard uncertainty of $0.038 \mathrm{~mm}$ in displacement. Then, the expanded measurement uncertainty for a confidence level of approximately $95 \%(k=2)$ is $0.076 \mathrm{~mm}$.

According to equation (14), the uncertainty of measurement of the maximum pressure $\left(P_{\max }=338.12 \mathrm{MPa}\right)$ could be calculated as follow:

$$
\begin{aligned}
u_{B}^{2}\left(P_{\max }\right)= & \left(\frac{\partial P_{\max }}{\partial x_{\max }}\right)^{2} u_{x_{\max }}^{2}+\left(\frac{\partial P_{\max }}{\partial A}\right)^{2} u_{A}^{2} \\
& +\left(\frac{\partial P_{\max }}{\partial V_{0}}\right)^{2} u_{V_{0}}^{2} .
\end{aligned}
$$

Moreover, the measurement uncertainty of the maximum pressure given by the SUT is estimated, based on the characteristics of the measuring chain components (technical documents and last calibration certificates of pressure transducers, charge amplifier and DAQ). Table 3 gives 
Table 2. Measurement uncertainty of the reference pressure by HSC.

\begin{tabular}{lc}
\hline Sources & $\begin{array}{c}\text { Contribution to } \\
\text { uncertainty }(\mathrm{MPa})\end{array}$ \\
\hline Displacement & 2.3762 \\
Piston section & 0.79 \\
Initial volume & 0.8237 \\
Combined-standard uncertainty & 2.63 \\
Expanded uncertainty $(k=2)$ & 5.2 \\
Relative uncertainty & $1.55 \%$ \\
\hline
\end{tabular}

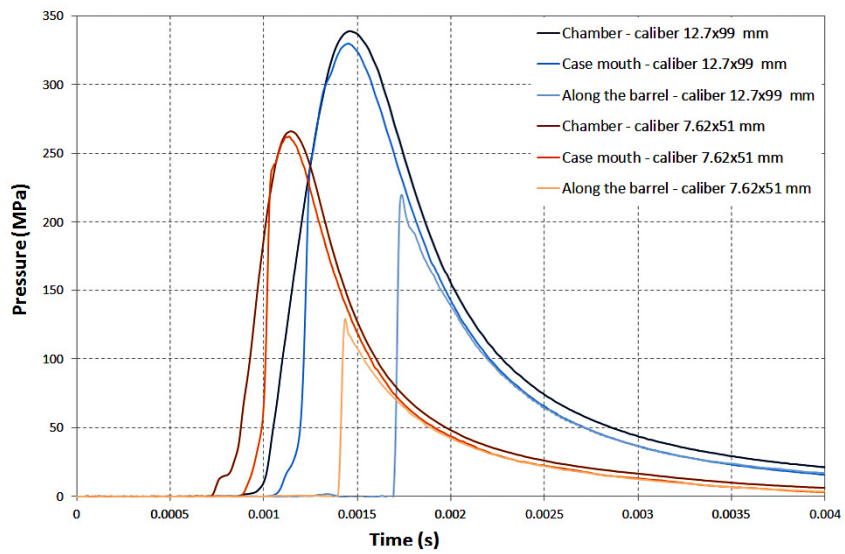

Fig. 17. Typical pressure curves of small calibers $12.7 \times 99 \mathrm{~mm}$ and $7.62 \times 51 \mathrm{~mm}$ with different measurement methods.

the contribution to uncertainty of each device and the resulting relative pressure uncertainty of the transducer under test.

According to Table 3, the measurement uncertainty of the SUT is arround $1.85 \%$. The combination of this uncertainty and the one of the reference pressure assessed in Table 2, gives the uncertainty of calibration which was evaluated to be around $2.40 \%$.

\section{A practical method for high-pressure dynamic calibration}

The peak value of the ballistic pressure inside a gun barrel, when an ammunition is fired, depends closely on the measurement method [19]. Given that the peak pressure and its uncertainty are the two most requested parameters, the fast changes of the ballistic pressure should be taken into account and performed with pressure transducers able to do it faithfully. Figure 17 permits to highlight the difference in pressure curve patterns depending on the caliber and the measurement method.

The proposed calibration method falls within this optic. The reference dynamic pressure was generated by impacting the piston in contact with an oil-filled cylindrical chamber by strikers of known masses and accelerated to the impact velocities by an air gun. The aim of the experimental setup is to work out a system which may generate known hydraulic pressure pulses with high-accuracy and controlled uncertainty. It permits obtain a frequency

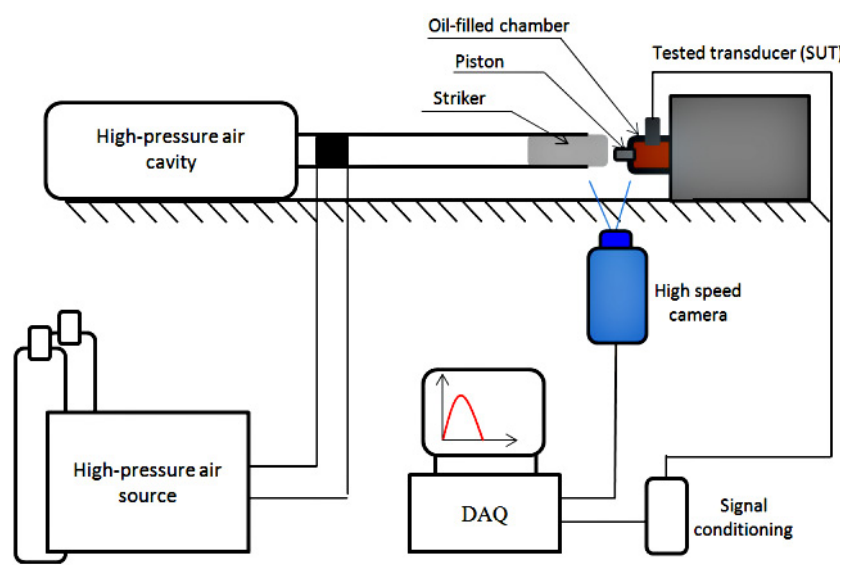

Fig. 18. The experimental set-up of dynamic pressure generator.

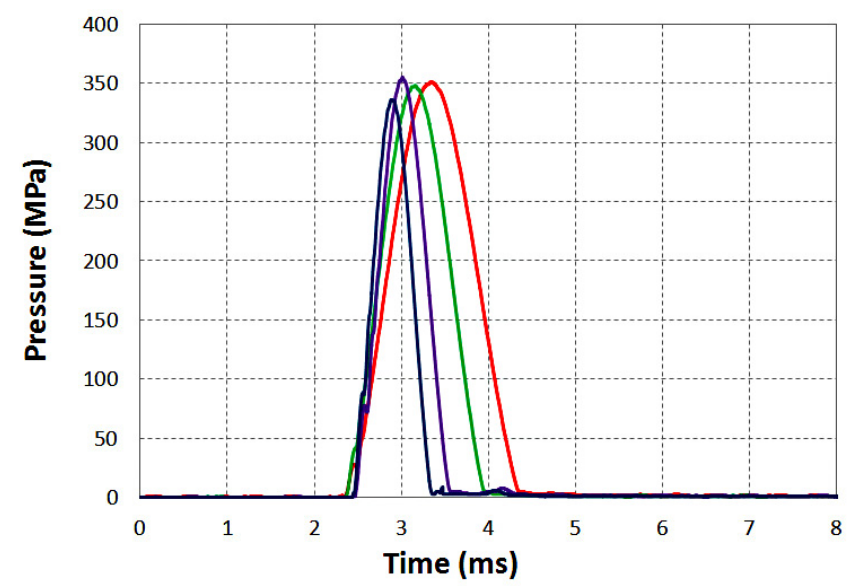

Fig. 19. The generated pressure pulses.

bandwidth at the range of $\mathrm{kHz}$ in which the dynamic behavior of the transducer can be investigated. The calculation of the reference pressure was based on the measurement of the displacement of the piston by a HSC as it was explained for drop-weight pressure pulse generator.

A device based on the firing of a projectile which hits a piston in contact with a compression chamber was developed at the Combat Systems Testing Activity (CSTA) in Maryland in the United States of America [20]. The use of this method is restricted because a projectile is fired during the process which requires extensive safety provisions. Therefore, it cannot be available in most laboratories.

Figure 18 gives an overview on the experimental setup which consists of four major components: a lunching device, an oil-filled compression chamber, a displacement measuring device (HSC) and a data acquisition and evaluation unit (DAQ). The strikers of different masses and velocities were launched by a sudden release of the compressed air in a gas gun and accelerated in a long barrel until impacting the piston.

As shown in Figure 19, the change of striker mass and velocity allows to obtain pressure pulses with different amplitudes and widths. A pressure pulse with peak value of $500 \mathrm{MPa}$ and width of $0.4 \mathrm{~ms}$ can easily be generated. 
Table 3. Measurement uncertainty of the pressure transducer.

\begin{tabular}{lcccc}
\hline Sources & Type & Value & Distrib. & $\begin{array}{c}\text { Contribution } \\
\text { to Uncertainty (MPa) }\end{array}$ \\
\hline Transducer & & & & \\
\hline Linearity & $\mathrm{B} 1$ & $0.14 \%$ & $\sqrt{3}$ & 0.2705 \\
Hysteresis & $\mathrm{B} 2$ & $1 \%$ & $\sqrt{3}$ & 1.9318 \\
Temperature sensitivity & $\mathrm{B} 3$ & $0.02 \% /{ }^{\circ} \mathrm{C}$ & $\sqrt{8}$ & 0.3691 \\
\hline Charge amplifier & & & & \\
\hline Linearity & $\mathrm{B} 4$ & $0.16 \%$ & $\sqrt{3}$ & 0.3091 \\
Resolution & $\mathrm{B} 5$ & 0.5 & $\sqrt{3}$ & 0.0236 \\
Precision & $\mathrm{B} 6$ & $0.5 \%$ & $\sqrt{3}$ & 0.9659 \\
Zero point deviation & $\mathrm{B} 7$ & $0.6 \mathrm{mV}$ & $\sqrt{3}$ & 0.0116 \\
Output Interference & $\mathrm{B} 8$ & $1.5 \mathrm{mV}$ rms & $\sqrt{3}$ & 0.0410 \\
Gain accuracy & $\mathrm{B} 9$ & $0.1 \%$ & $\sqrt{3}$ & 0.1932 \\
\hline Data acquisition & \multicolumn{3}{c}{} & 0.0059 \\
\hline Resolution & $\mathrm{B} 10$ & 0.5 & $\sqrt{3}$ & 0.7727 \\
Offset accuracy & $\mathrm{B} 11$ & $0.4 \%$ & $\sqrt{3}$ & 1.2557 \\
DC accuracy & $\mathrm{B} 12$ & $0.65 \%$ & $\sqrt{3}$ & 1.3523 \\
AC amplitude accuracy & $\mathrm{B} 13$ & $0.7 \%$ & $\sqrt{3}$ & 0.0657 \\
RMS noise & $\mathrm{B} 14$ & $0.03 \%$ & $\sqrt{3}$ & 0.73 \\
Repeatability error $(10$ measures, CL of $95 \%)$ & $\mathrm{A} 1$ & $0.34 \%$ & - & \\
Combined-standard uncertainty & $3.1 \mathrm{MPa}$ & & & \\
Expanded uncertainty $(k=2)$ & $6.2 \mathrm{MPa}$ & & & \\
Relative uncertainty & $1.85 \%$ & & &
\end{tabular}

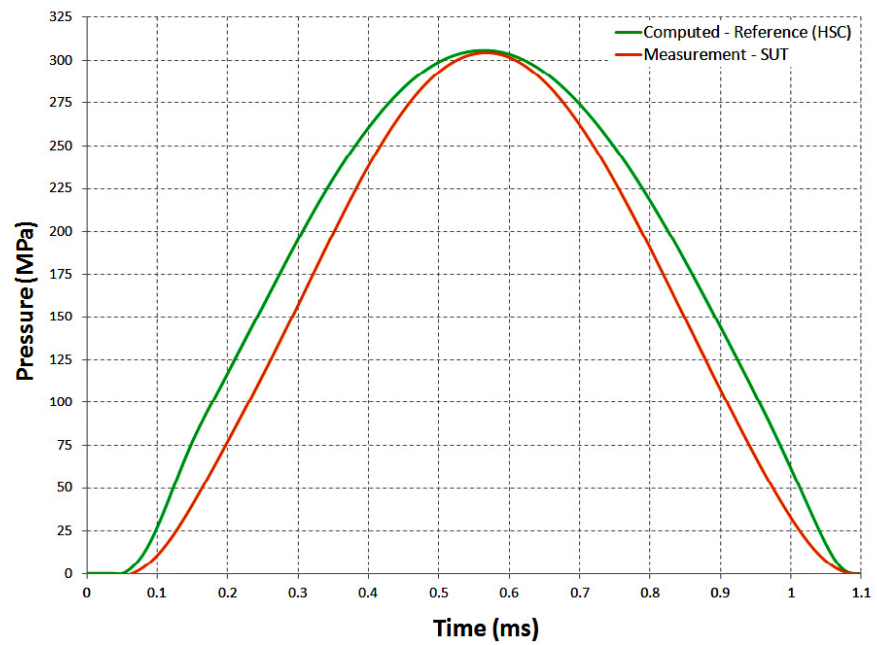

Fig. 20. Reference and SUT pressure pulses.

Using the model described by equation (10), the reference pressure was computed from the displacement of the piston/striker given by HSC and compared to the measured one (SUT) as illustrated in Figure 20.

\section{Conclusion}

The calibration of piezoelectric pressure transducers is paramount in ballistics. This operation should be achieved taking into account the characteristics of the mechanical quantity which must be measured. The presented study aimed at setting up a method for calibration under dynamic conditions of these transducers. The displacement measurement with a high speed camera is a promising approach for calculating the reference pressure. The measurement uncertainty was assessed to be around $2.40 \%$. Moreover, in order to perform calibration in greater range of frequencies, an approach based on the launching of strikers from an air gun was presented. This method is simple to set up and easy to use. The variation of the mass and velocity of the striker allows to produce pressure pulses of different amplitudes and frequencies. The first results show that a frequency bandwidth in the order of $\mathrm{kHz}$ could be reached. Therefore, this method could be a promising way for the dynamic calibration of high pressure transducers.

\section{References}

1. KIAG, Technical documents, KISTLER Instrumente AG (KIAG). Available at http://www.kistler.com

2. V.E. Bean, Dynamic pressure metrology, Metrologia 30, 737-741 (1993)

3. J.P. Damion, Means of dynamic calibration for pressure transducers, Metrologia 30, 743-746 (1993)

4. J.L. Schweppe, L.C. Eichenberger, D.F. Muster, E.L. Michaels, G.F. Paskusz, Methods for the dynamic calibration of pressure transducers, NBS Monograph 67 (1963)

5. ASME, A Guide for the dynamic calibration of pressure transducers, ANSI B88.1-1972, USA, 1995

6. ISA, A guide for the dynamic calibration of pressure transducers, ISA-37.1601-2002, USA, 2002 
7. J. Winkler, Quality improvement in piezoelectric pressure measurements by applying advanced calibration methods, 4 International Symposium on Ballistic Measurement, Switzerland, September 25-29, 1989

8. J.P. Damion, Capteur de pression de référence pour l'étalonnage dynamique, re Congrès International de Métrologie, France, 1995

9. C. Cater, Calibration of piezoelectric sensors for combustion pressure analysis, KIAG, 2006

10. M. Kobusch, T. Bruns, E. Franke, Challenges in practical dynamic calibration, AMCTM, France, June 23-25, 2008

11. J. Lally, D. Cummiskey, Dynamic pressure calibration, PRS-TN-15-0205, PCB Piezotronics, 2005

12. C. Bartoli1, M. F. Beug, T. Bruns, C. Elster, T. Esward, L. Klaus, A. Knott, M. Kobusch, S. Saxholm and C. Schlegel, Traceable dynamic measurement of mechanical quantities: objectives and first results of this european project, Int. J. Metrol. Qual. Eng. 3, 127-135 (2012)

13. T. Bruns, E. Franke, M. Kobusch, Linking dynamic to static pressure by laser interferometry, Metrologia 50, 580-585 (2013)
14. Y. Zhang, J. Zu, H.Y. Zang, Dynamic calibration method of high-pressure transducer based on quasi-delta function excitation source, Measurement 45, 1981-1988 (2012)

15. Photron, FASTCAM SA-5 specifications. Available at http://www.photron.com

16. C. Robbe, N. Nsiampa, A. Oukara, A. Papy, Quantification of the uncertainties of high-speed camera measurements, Int. J. Metrol. Qual. Eng. 5, (2014)

17. NI, Technical documents of LabVIEW, National Instruments (NI). Available at http://www.ni.com/ labview

18. AFNOR, Norme AFNOR NF ENV 13005: Guide pour l'expression de l'incertitude de mesure, AFNOR, Août 1999

19. L. Elkarous, F. Coghe, M Pirlot, J-C Golinval, Experimental techniques for ballistic pressure measurements and recent development in means of calibration, J. Phys.: Conf. Ser. 459, 012048, 2013

20. A.A. Juhasz, C.D. Bullock, D.H. Newhall, Calibration of ballistic pressure transducers, Measurement and Control 146, 123-127 (1991) 